\title{
Rheological Properties of Traditional Balsamic Vinegar: New Insights and Markers for Objective and Perceived Quality
}

\author{
Pasquale M. Falcone ${ }^{a^{*}}$, Elisa Sabatinelli ${ }^{\mathrm{a}}$, Federico Lemmetti $^{\mathrm{b}}$, And PaOlo \\ GIUDICI $^{b}$ \\ ${ }^{\text {a }}$ University Polytechnical of Marche - Department of Agricultural, Food and Environmental Sciences, Brecce \\ Bianche 2 - 60128 Ancona, Italy \\ ${ }^{\mathrm{b}}$ University of Modena and Reggio Emilia, Unimore Microbial Culture Collection - Department of Life \\ Sciences - Via Giovanni Amendola 2 - 42122 Reggio Emilia, Italy \\ ${ }^{*}$ Corresponding author \\ pm.falcone@univpm.it \\ TEL: +390712204138
}

Received: 24 October 2016; Published online: 18 April 2017

\begin{abstract}
The molecular structure of Traditional Balsamic Vinegar (TBV) undergoes shear-induced and timedependent jamming transitions due to the high solute concentration and self-assembling phenomena of high molecular size melanoidins with very-long relaxation times (12 years at least or more than 25). The purpose of this work was to perform a descriptive and quantitative evaluation of relationships between rheological properties, vinegar composition, and perceptual assessment of sensory properties according to the official sensory procedure. With this aim, vinegars having quality traits matching legal requirements for the PDO designation were analyzed for their reducing sugars (glucose and fructose), volatile acidity, fixed acidity, $\mathrm{pH}$, Brix degree, and density as well as for their flow behaviour and dynamic viscosity over a wide range of shear rates. Results showed that flow behaviour of TBV was affected by jamming properties over wide-scale ranges of shear rate producing flow instability below a shear rate of $60 \mathrm{~s}^{-1}$. Homogeneous, continuous flow was found at medium-high shear rates with thickening and/or thinning traits. A common onset for the structure scaling was mathematically estimated to occur close to when the density was $1.32 \mathrm{gmL}^{-1}$. Comparative analysis of rheological, compositional and sensory properties suggested that the colloidal jamming of the vinegar melanoidins dominated the total olfactive and gustative stimuli, and determined the classification of the vinegars that had a higher dynamic viscosity but more homogeneous flow as being of the highest commercial quality category. A robust statistical model was proposed encoding for the top-down decision-making process for quality assignment according to the official sensory procedure, using composition and flow properties as predictor variables.
\end{abstract}

Keywords: Jamming transition; Melanoidins; Flow properties; Viscosity; Perceptual assessment of sensory properties; POD disciplinary

\section{Introduction}

Traditional Balsamic Vinegar (TVB) of Modena (TBVM) and Traditional Balsamic Vinegar of Reggio Emilia (TBVRE) are two high-value Italian aged vinegars produced in the Modena and Reggio Emilia provinces (northern Italy), for which the European Council has granted PDO status, i.e. protected geographical origin and designation (Gazzetta Ufficiale della Repubblica Italiana 124/2000; Regulation EC 813/2000). Two independent authorities certify the age and 
quality requirements imposed by the regulation according to the official taste procedure, which is based on the sensorial ranking of a series of coded visual, olfactory, gustatory and olfactorygustatory attributes and on the instrumental measurement of density and titratable acidity. Originally, these artisanal products were only available to Italians on higher incomes, but now both TBVs are gaining worldwide great recognition for their sensorial, nutritional, health properties (Tagliazucchi, Verzelloni, \& Conte, 2010; Lemmetti, Falcone, \& Giudici, 2013; Lemmetti, Solieri, Bonciani, Zanichelli, \& Giudici, 2014). TBV is very labour-intensive to produce and it is the only vinegar around the world that is produced starting from cooked grape juice only. The historical procedure requires that the grapes must be cooked with a direct flame in open vessels, then the cooked must is fermented and oxidized by autochthonous microorganisms and, finally, the resulting vinegar ages within casks of decreasing volumes made of different woods (oak, mulberry, chestnut, cherry, juniper, ash, and acacia). While it ages over decades and gradually evaporates under seasonal temperature and relative humidity, the vinegar is transferred to successively smaller casks becoming sweet, concentrated, flavoured and viscous. Time plays a central role in the overall production of TBV, requiring a legal minimum for the aging period of 12 to 25 years at least, which promotes profound changes in composition (Plessi, Monzani, \& Coppini, 1988; Cocchi et al., 2006), volatile compounds (Duran Guerrero, Chinnici, Natali, Natera Marin, \& Riponi, 2008), rheological (Falcone, Chillo, Giudici, \& Del Nobile, 2007, 2008) and sensory properties (Giudici, Falcone, Scacco, \& Lanza, 2009). The classes of the major components in TBV include glucose, fructose, acetic, gluconic, malic, tartaric, and succinic acids. The classes of the minor compounds refer mainly to volatile compounds, polyphenols and melanoidins, a compositionally and structurally heterogeneous blend of sugar/phenol-derived polymeric melanoidins showing antioxidant and radical scavenging properties (Verzelloni, Tagliazucchi, \& Conte, 2007). From a physical perspective, TBV has been recognized as a soft material showing different flow behaviours spanning from Newtonian to shear thinning, shear thickening and viscoelastic regimes under controlled shearing conditions. Using different and complementary techniques, the major contribution of the polymeric melanoidins to local jamming phenomena forming metastable structures has been recently recognized throughout the entire aging process (Falcone \& Giudici, 2008; Falcone, 2010; Falcone, Boselli, \& Frega, 2011, 2012). "Jamming" is a term widely used in the science of food structure for soft materials (Mezzenga, Schurtenberger, Burbidge, \& Michel, 2005; Ubbink, Burbidge, \& Mezzenga, 2008) in order to describe how their constituents may result metastable structures that reach a minimum free energy as well as how some external parameters including density, temperature, and mechanical stress can influence such out-of-equilibrium structures by enhancing, reducing or eliminating the ability to flow (Liu \& Nagel, 1998; Cates, Wittmer, Bouchaud, \& Claudin, 1998; Trappe, Prasad, Cipelletti, Segre, \& Weitz, 2001; Liu \& Nagel, 2001). Falcone et al. (2012) attributed vinegar jamming to the selfassembly properties of vinegar melanoidins due to their molecular properties: (i) the wide range of molecular size distribution that spreads from $200 \mathrm{Da}$ to beyond $2000 \mathrm{kDa}$ (ii) the distribution on the chain backbone of attractive net charges and bulky aromatic phenolic residues. Other compositional factors were also hypothesized to play a role in the extent of colloidal jamming in longaged vinegars: $\mathrm{Fe}$ and $\mathrm{Mg}$ content were higher and $\mathrm{pH}$ was lower in jammed fractions of the vinegar rather than flowing ones.

From a sensory perspective, the personal ability of trained judges to classify vinegars according to their sensing and perceptual assessment of vinegar properties is first to develop a codified concept of the visual, taste, olfactory, and trigeminal properties. Then they are required to evaluate such vinegar properties in an independent way under a series of mechanical stimuli the most of which arise into the mouth before, during and after swallowing. However, the independence of the stimuli assessment is not realistic in literal sense due to the simultaneous nature of human perceptions of chemical and flavour resulting in synergistic or masking effects depending on mechanical stimuli. In other food beverages of similar composition and at the same temperature, 
New Quality Markers for Traditional Balsamic Vinegar $\mid 97$

the viscosity affects flavour release (Cook, Hollowood, Linforth, \& Taylor, 2003; Hollowood, Linforth, \& Taylor, 2002; Vingerhoeds, de Wijk, Zoet, Nixdorf, \& van Aken, 2008), and determines threshold for sweetness and creaminess perception (Zahn, Hoppert, Ullrich, \& Rohm, 2013). Thickness is the texture attribute worldwide used to describe perceptual assessment of the flow behaviour of a food beverage. Thickness can be assessed in the mouth by judging the resistance of the sample to flow, the rate of flow, or the amount of force needed to translocate the fluid in the mouth (Akhtar, Stenzel, Murray, \& Dickinson, 2005). For a more objective evaluation, the simple approach of considering the tongue-palate as two plates has been used for a long time as a basis for attempting to associate the thickness of food materials to the dynamic viscosity measured in the cone-plate or cylindrical geometry of a rheometer (Chen \& Engelen, 2012). Thus, the dynamic viscosity of a homogeneous fluid can be interpreted as a macroscopic expression of the friction of the constituents opposing the flow under controlled shearing conditions. However, it is important to have knowledge about the smallest differences in dynamic viscosity that the human senses can discriminate: the same thickness does not necessarily mean the same dynamic viscosity (Camacho, Dop, de Graaf, \& Stieger, 2015).

The purpose of this work was to perform a descriptive and quantitative evaluation of relationships between rheological properties, vinegar composition, and perceptual assessment according to the official taste procedure.

\section{Materials and Methods}

\subsection{Sourcing of vinegar samples}

In order to characterize vinegars for their rheological properties, all vinegar samples were randomly collected from different vinegar farms with the aim of having a broad distribution of product composition, structure and flow behaviour. However, only vinegars having quality traits matching legal requirements for the PDO designation were analysed for their composition and rheological properties. Besides the requirements for the production process, the regulation specifies the chemical-physical parameters and sensory traits, indicating as compulsory their perceptual assessment before submitting vinegar lots for PDO authorization. The official sensory procedure imposes firstly the trained evaluation of visual traits, followed by olfactory perception and finally tasting, i.e. viscosity, colour and clearness for the "visual traits"; delicacy, defectiveness, persistence and acidity for "olfactory traits"; fullness, maturity, harmony and acidity for "gustatory traits". Perceptual discrimination and classification of all these organoleptic properties arise from a human complex decision-making process driven by sensory and psychological factors that are expressed by the judges as quantitative and cumulative scores. Official sensory panels award a final score, which determines the commercial quality and price categories: the Consortium of the ABTRE discriminate three quality levels ("aragosta", "argento", and "oro"). Trained judges individually taste and express their evaluation filling a structured questionnaire: each sensory descriptor has a pre-printed ranking of scores and the panellist has to indicate the score that best matches his or her perception of intensity.

In this work a trained panel composed of five members from the official list of "ABTRE expert tasters" registered in the Chamber of Commerce of Reggio Emilia was asked to analyse more than one thousand TBVRE vinegars for their visual, gustatory and tasting traits according to the official sensory procedure as well as for ranking them on the basis of their perceptual intensity. For these vinegars the producers claimed an aging period ranging from 12 to 25 years, which granted them the participation in the historical public competition known as "Palio Matildico" held at 2014 in Arcevia (Reggio Emilia, Italy). Only 100 vinegars received the judgment "vinegar matching law requirements for the PDO designation" from which a subsets of 20 vinegars was assigned to the quality level "oro", while the remaining 80 vinegars were assigned to the "aragosta" or "argento" ones. In this work "F" is the label assigned to those vinegars that judges recognized as to "aged vinegars of very-high sensory quality" and corresponding to the quality level "oro"; while " $\mathrm{R}$ " is the label assigned to 
98 Falcone et al.

those vinegars that judges recognized as to "aged vinegars of acceptable sensory quality" and corresponding to the quality level "aragosta" or "argento". All of samples were further coded using random alpha numeric labels " $\mathrm{F}[\mathrm{n}]$ " or "R[n]" with $[\mathrm{n}]$ the three digit part of the code.

\subsection{Composition properties}

Vinegars samples were analysed for glucose and fructose (the sum of which is indicated as "sugars"), volatile acidity ( $\mathrm{AcVol})$, titratable acidity (AcTit), fixed acidity (AcFix), pH, specific weight (density) and Brix degree (Brix). Density was measured with a hydrostatic balance (Densimat, Gibertini, Italy) and Brix with a refractometer (2WA, Alessandrini, Italy); they were used to cumulatively estimate the overall concentration of the vinegar. Enzymatic kits (Roches Co. Darmstad, Germany) coupled with a spectrophotometer UV-Vis (V-550, Jasco, Japan) were used to determine the sugars and acetic acid concentration $(\% \mathrm{w} / \mathrm{w})$, the latter was assumed equivalent to the AcVol. AcTit was determined with titration method using $\mathrm{NaOH}$ and phenophthalein indicator to stop neutralization at $\mathrm{pH} 7$. AcFix was calculated as difference between AcTit and AcVol. A digital pH-meter (model 8417, Hanna Instruments, Padova, Italy) was used to measure the $\mathrm{pH}$ value after dilution with HPLCgrade water in the ratio 1 to 10 by weight.

\subsection{Rheological experiments}

Flow behaviour was investigated with a rheometer (Rheomat RM180, Maple Instruments) equipped with concentric cylinders complying to the size requirements of DIN 53019 (or ISO 2555) as the measuring system. Shear stress data were acquired by performing steady-state shearing tests based on the step changes of single point measurements, i.e. torque required to hold for a given time the vinegar sample under shearing at a given shear rate (with an accuracy of the rotation speed was $\pm 0.5 \%$ of the actual value). The dynamic viscosity was calculated as the ratio between the shear stress and steady-state shear rate. Flow experiments were carried out within measuring tubes immersed in a water bath for temperature control $\left(25 \pm 0.5{ }^{\circ} \mathrm{C}\right)$. Samples were loaded into the system, allowing them to come out gently from a vial reservoir. The waiting time required establishing a baseline shear history was fixed at 5 minutes.

\subsection{Flow curve analysis and modelling}

Stress data were analysed as a function of shear rate by means of a theoretical model. Software package Pro Fit ver. 6.2.16. (Quantum Soft Inc. 2015, Uetikon am See, Switzerland) was used to run the robust algorithm for non-linear regression of the models. For comparative purposes, fitting parameters were estimated together with their confidence limits $(95 \%, \mathrm{p}<0.05)$. Error analysis was performed accounting for $0.5 \%$ on the actual value of shear rate and running the fitting procedure 500 times so that descriptive statistics were finally calculated from the distributions of the parameters. The goodness-of-fit was evaluated by calculating the mean relative error according to Boquet, Chirife, and Iglesias (1978), using expression (1).

$$
E=\frac{100}{N} \times \sum_{i=1}^{N} \frac{\mid\left(y_{i}^{o b s}-y^{c a l c} \mid\right.}{y^{c a l c}}
$$

Where $\mathrm{y}^{\text {obs }}$ are the experimental data, $\mathrm{y}^{\text {calc }}$ are the predicted data; $\mathrm{N}$ is the number of data points

\subsection{Statistical Analysis}

Descriptive, principal component and tree classification analyses were performed using the software package STATISTICA ver. 10 (StatSoft Inc. 2011, Tulusa, USA) accounting for composition and rheological data as input variables with and without a grouping variable in order to perform supervised and unsupervised analysis of data structure.

Unsupervised recognition methods are helpful when the researcher wishes to discover the class membership of a sample, while supervised pattern recognition methods are helpful to discover the sample properties that distinguish sample classes. In this case, the algorithm requires 
New Quality Markers for Traditional Balsamic Vinegar |99

class information to be input. It is well known that conventional principal component analysis (PCA) can be performed as a classical data analysis method providing a dimensionality reduction into data set able to capture the maximum variability in the data, and can be viewed as modelling the covariance structure of the data. However, the effectiveness of conventional PCA is limited to unsupervised problems, making it impossible to guide the algorithm toward the modes of variability that are of particular interest. In the present work, we were interested to classify vinegars on the basis of their composition and rheological properties, attempting to project the explanatory variables along directions that were related to a desired response variable, i.e. $\mathrm{R}$ and F quality category. This goal is not achieved by using the directions that have maximum variation. Thus, in order to discover composition and rheological properties able to classify vinegars belonging the quality categories (i.e. $\mathrm{F}$ or $\mathrm{R}$ ), we performed a "supervised principal component analysis" using quality category as grouping variable and all composition and rheological properties as independent variables. The latter were used as input for supervised PCA and chosen from those showing the most statistical significance in the Kolmogorov-Smirnov two-sample test. The latter test was compared to standard descriptive statistics based on theoretical symmetrical distribution of data.

Without any a priori hypotheses about how many independent quality categories as well as about what and how many vinegars may have belonged to each of them, unsupervised classification of the vinegars was performed by means of tree classification analysis (TCA) for which all composition and rheological properties were used as predictor variables. Classification, splitting and validation rules were used to examine their effects one at a time, rather than just all at once. The unsupervised top-down classification process started by dividing the initial set of vinegars into subsets in a hierarchical manner, using the best splitting value test for one composition or rheological property at time used as target variable. Such recursive partitioning was completed when splitting no longer added value to the predictions. The classification rule was to assign membership of vinegars to the $\mathrm{F}$ or $\mathrm{R}$ cat- egory with the same accuracy regardless of the frequency really assigned by the judges. Vinegar misclassification was evaluated by comparing the unsupervised vinegar classification and the assignment of them into the two quality categories by trained judges based on their perceptual assessment of their sensory properties. In order to minimize misclassification error, only one case (one vinegar sample) was imposed as a stopping rule at terminal nodes. The initial data set was divided into the training and validation dataset and a V-fold cross validation algorithm (with V equal to 60 ) was used for validation purposes.

\section{Results and Discussion}

\subsection{Theoretical analysis of shear dependence of the flow}

The molecular size and molecular size distribution as well as the self-assembling and jamming properties of melanoidins present in long-aged traditional balsamic vinegars were discussed in previous works (Falcone \& Giudici, 2008; Falcone et al., 2012). To get a fundamental insight about the effect of jamming properties on the overall flow behaviour for the vinegar samples, flow curves were analyzed using a modified Krieger-Dougherty equation (Krieger \& Dougherty, 1959) for relative viscosity. Such a model provided valuable guidelines for understanding the role of the colloidal structure in flow behaviour as a function of the concentration of the attractive colloidal melanoidins dispersed into the vinegar bulk. The functions (2) and (3) were fitted to the experimental data. The goodness-of-fit for eq. 2 and eq. $3(\mathrm{E} \%<9.75 \%)$ indicated that TBVRE behaved as a colloidal dispersion of attractive melanoidins for which the onset for the structure scaling signing the transition from Newtonian to non-Newtonian flow regime was close to the relative density of $1.32 \mathrm{gmL}^{-1}$ (Figure 1).

As indicated by the apparent discontinuity of the shear stress profiles, TBVRE showed intermittent flow at low shear rates (mainly under 60) followed by continuous flow at higher shear rates. Figure $2 \mathrm{a}$ and $2 \mathrm{~b}$ show the two flow regimes for the vinegar samples "R376" and 
"F227" as examples for quality categories R and $\mathrm{F}$, respectively. According to a scheme generally recognized for other soft jammed systems such as colloidal, micellar and polymeric suspensions that show typical intermittent flow behaviour (Coussot \& Ovarlez, 2010; Holmes, Callaghan, Vlassopoulos, \& Roovers, 2004; Schall \& van Hecke, 2010; Princen \& Kiss, 1989), we attempted to interpret the flow instability in the vinegars from a physical perspective. With this aim, we attributed the observed intermittent flow to the progressive evolution of the viscoelastic structure of colloidal melanoidins under mechanical shearing. Recent studies showed that long-aged traditional balsamic vinegars with high Brix values (over $75^{\circ} \mathrm{Bx}$ ) show yield stress and viscoelastic behaviour under sweep experiments from 10 to $200 \mathrm{~Hz}$ (Falcone et al., 2012). With this in mind, two structuring, competing phenomena were hypothesized, one on them becoming dominant depending on the flow conditions: (i) reversible restructuration at long-range scales of the locally-discontinuous network of colloidal melanoidins due to the inter-chain entanglements (elastic contribution to colloidal jamming), that may oppose the starting flow (yield stress) and/or the flow at lower shear rates (intermittent flow behaviour) and (ii) irreversible restructuration at shortrange scales of viscous local bonds through local reptation of the chain backbone of polymeric melanoidins(viscous contribution to sub-colloidal jamming), that may result in homogeneous flow regime with shear-thinning or shear-thickening behaviour depending on the effective hydrodynamic interactions. Discontinuous colloidal networks deform under shear until they break rapidly when the elastic stress reaches maximum critical values. As elastic bonds are broken and reformed reversibly, stress changes abruptly. As there is no more elastic interaction among dispersed colloids, the stress needed to maintain flow is only the simple viscous one and changes may occur due only to the shear-thinning or shear-thickening phenomena.

$$
\frac{\eta_{\text {low }}}{\eta_{m}}=\left[1-\frac{d}{d_{\max }}\right]^{-\eta_{\text {in }} \times d_{\max }}
$$

$$
\frac{\eta_{h i g h}}{\eta_{m}}=\left[1-\frac{d}{d_{\max }}\right]^{-\eta_{i n} \times d_{\max }}
$$

Where $\eta_{\text {low }}$ and $\eta_{\text {high }}$ are the apparent viscosity measured at $60 \mathrm{~s}^{-1}$ (where the intermittent flow starts to disappear) and at $600 \mathrm{~s}^{-1}$ (where Newtonian flow starts to be recovered); $\eta_{m}$ is the vinegar bulk viscosity which was calculated using the $2^{\text {th }}$-order equation provided by Falcone et al. (2007) for aqueous solutions of glucose, fructose and acetic acid (the main vinegar constituents simulating the TBV composition); $d$ is the relative density used here to represent the overall concentration of structure-forming constituents including isolated/discontinuous aggregates of colloidal melanoidins; $d_{\max }$ represents the maximum packing fraction of structureforming constituents due to the formation of a continuous network structure among attractive colloidal melanoidins; $\eta_{i n}$ is the intrinsic viscosity, a measure of the extent of jamming at a colloidal scale $\left(\mathrm{d}_{\max }\right.$ and $\eta_{i n}$ were treated as a fitting parameter). When the vinegar viscosity is equal to that of its bulk medium, then relative viscosity $\left(\eta_{\text {low }} / \eta_{m}\right.$ or $\left.\eta_{\text {high }} / \eta_{m}\right)$ is equal to 1 ; an increase of relative viscosity must be attributed to an increase of the colloids concentration and/or to the strength of their interactions.

\subsection{Perceptual probing of jamming properties}

The visual perception of the characteristic flow behaviour under gravity is considered historically a key point to meet quality requirements in terms of vinegar thickness as imposed by the official regulations for TBVRE. The term adopted to indicate the optimal thickness is "syrup flowiness" or "flow syrupiness", both resembling the flow of concentrated syrups. Trained judges evaluated the vinegar thickness by inspecting visually the pouring speed from a glass bottle and the ability to leave a film transparent to the light of a candle. It is worth noting that such a visual method cannot discriminate small differences in kinematic viscosity, i.e. the resistance to flow per unit of specific weight. Small differences in pouring speed and time of observation strongly affect visual perception of vine- 
New Quality Markers for Traditional Balsamic Vinegar |101

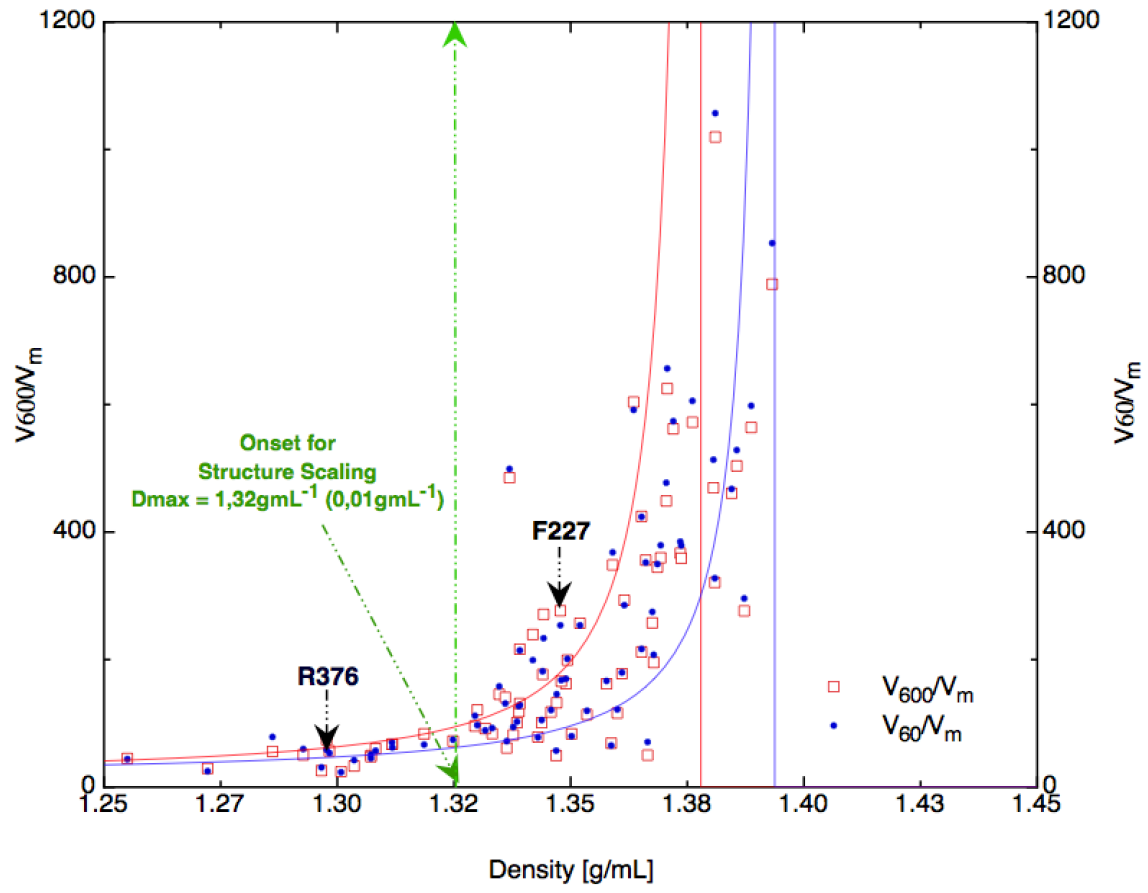

Figure 1: Shear-limited viscosity data fitted with the modified Krieger-Daugherty model. TBVRE behaved as a colloidal dispersion of melanoidins with the onset for solute concentration signing transition from Newtonian to non-Newtonian flow regime

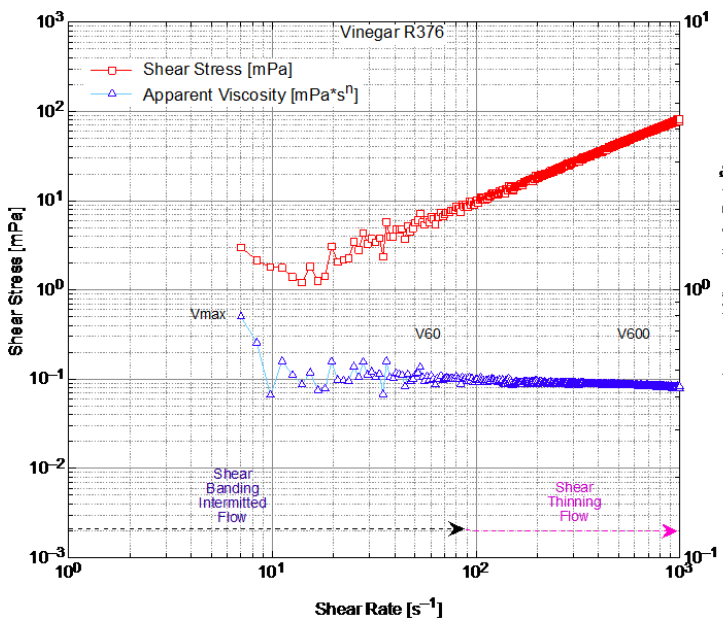

(A)

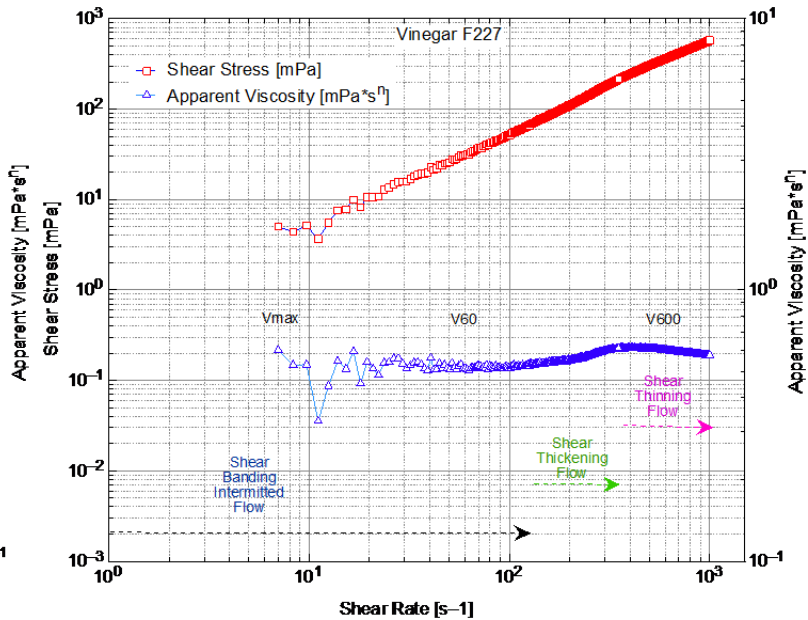

(B)

Figure 2: Shear-induced transition from intermittent to homogeneous flow regime: (a) the vinegar R376 belonged to the quality category R, i.e. "aged vinegars of acceptable sensory quality"; (b) the vinegar F227 belonged to the quality category F, i.e. "aged vinegars of very-high sensory quality" 
gar thickness. Moreover, kinematic viscosity may vary with the flow rate for vinegars showing jamming properties and non-Newtonian behaviour, dynamically affecting the strength of the film and transparency to light. Differences in concentration of visible light-absorbing molecules in vinegar bulk may also affect transparency at the same vinegar thickness or kinematic viscosity.

With this in mind, it was reasonably hypothesized that colloidal and jamming properties together with the non-Newtonian behaviour might affect in a synergistic or masking way not only vinegar thickness under gravity as evaluated by visual inspection but also stress stimuli and the way they are perceived during vinegar sensing so determining the final quality score. As a consequence, in this work we decided to examine the shear stress developed over a wide range of controlled shear rates, i.e. $1-1000 \mathrm{~s}^{-1}$ against the overall perceptual score used to assign vinegars along with two different commercial categories. The shear rate ranging from $50 \mathrm{~s}^{-1}$ to $200 \mathrm{~s}^{-1}$ includes those experienced by a Newtonian liquid pouring from a bottle (Gebhard, 2000). However the vinegars under investigation showed Newtonian behaviour only after $600 \mathrm{~s}^{-1}$ thus the kinematic viscosity was evaluated at this targeted shear rate with the purpose of simulating mechanical stimuli probed by visual inspection of the Newtonian flow under gravity. The shear rates ranging between $1 \mathrm{~s}^{-1}$ and $60 \mathrm{~s}^{-1}$ are long-term, even though they are the most frequent shear rate condition experienced by many fluid foods in the mouth during tasting (Wood, 1968; Shama, Parkinson, \& Sherman, 1973). Falcone et al. (2012) showed that the size of the colloids of vinegar melanoidins spreads in a wide range (from sub-colloidal to macroscopic) with the most frequent around $50-100$ microns. It happens that this size is similar to the size that humans can sense with the tongue, and thus they are expected to affect the stress stimuli during vinegar tasting.

As far as the shear forces experienced in this study are concerned, they varied between about $90 \mathrm{mN}$ and $500 \mathrm{mN}$ within the range of shear rates from $10 \mathrm{~s}^{-1}$ to $60 \mathrm{~s}^{-1}$ (Table 1 ). Such a range of shear forces includes force stimuli the human tongue can probe by mechanoreceptive afferents. Trulsson and Essick (1997) found that the lin- gual nerve contains 33 single mechanoreceptive afferents particularly innervating the tip of the tongue. Of these afferents, 22 were located at surface of the tongue and respond to extremely low mechanical forces, with force threshold range between $300 \mathrm{mN}$ and $2 \mathrm{mN}$. The remaining 11 afferent units are located in the tongue muscle mass and responded to high force threshold, i.e. $>4 \mathrm{mN}$. The superficial units were classified as rapidly adapting afferents to the stress stimuli: they respond both during the application and removal of, but not during maintenance of, stress stimuli on the receptive field. Among the deep afferent units, two type of slowly adapting responses were observed: one type was characterized by a pronounced sensitivity to force changes during the application and removal of the stress stimuli and an irregular static discharge during maintenance of the stimulus on the receptive field. In contrast, the other afferent units exhibit a weak sensitivity to force change, a highly regular static discharge, and spontaneous activity. Finally, these authors concluded that the superficial units encode vigorously when the tongue is moved to bring the receptive field into physical contact with other intraoral structures.

\subsection{Flow behaviour vs perception of colloidal jamming}

With the purpose of simulating key force stimuli experienced by tongue during vinegar tasting, the following two rheological descriptors were obtained from flow curves. The ratio $\mathrm{R}(1,2)$ was calculated by dividing the shear forces measured at $10 \mathrm{~s}^{-1}$ and $60 \mathrm{~s}^{-1}$, so that levels with a difference greater than 1 indicated changes in density heterogeneities at colloidal scale as induced by low-shear rates (yield stress or intermittent flow regime). Thus $R(1,2)$ was associated to the inmouth mechanical stimuli encoding for probing of the extent of jamming at colloidal scale. Furthermore, the ratio $\mathrm{R}(2,3)$ was calculated by dividing the shear forces measured at $60 \mathrm{~s}^{-1}$ and $600 \mathrm{~s}^{-1}$ so that levels greater than 1 indicated decreasing in density heterogeneities at sub-colloidal scale as induced by medium-high shear rates (homogeneous flow with shear-thinning effects). Levels of $\mathrm{R}(2,3)$ lower than 1 indicated increasing of local 
New Quality Markers for Traditional Balsamic Vinegar |103

Table 1: Distribution of shear stress values measured at $10 \mathrm{~s}^{-1}, 60 \mathrm{~s}^{-1}$ and $600 \mathrm{~s}^{-1}$

\begin{tabular}{ccc}
\hline & Mean $(*)$ & Std.Dev. \\
\hline Stress [mN] at $10 \mathrm{~s}^{-1}$ & $5,65[0.95-10.35]^{a}$ & 4,7 \\
Stress [mN] at $60 \mathrm{~s}^{-1}$ & $28,90[2.82-54.98]^{a}$ & 26,08 \\
Stress [mN] at $600 \mathrm{~s}^{-1}$ & $282,47[34.44-530.5]^{b}$ & 248,03 \\
\hline
\end{tabular}

$(*)$ Different letters indicate statistically significance difference between confidence limits

density heterogeneities as induced by mediumhigh shear rates (homogeneous flow with shearthickening effects). Thus $R(2,3)$ was associated to the in-mouth mechanical stimuli encoding for probing of the extent of jamming at sub-colloidal scale. A clear picture of vinegar distribution (Figure $3 \mathrm{a}$ and $3 \mathrm{~b}$ ) was obtained as a function of perceptual classification of sensory properties, accounting for kinematic viscosity measured at $600 \mathrm{~s}^{-1}$ (V600/Density) and the associated jamming descriptors $\mathrm{R}(1,2)$ and $\mathrm{R}(2,3)$. As can be inferred from data, the scale length of jamming clearly affected both the Newtonian flow under gravity and perceptual classification of the sensory properties. The closer the colloidal jamming extent, i.e. the value of $\mathrm{R}(1,2)$ was to 1 , the greater the kinematic viscosity. The greater the extent of jamming at sub-colloidal scale, i.e. the value of $\mathrm{R}(2,3)$ was far from 1 , the lower the kinematic viscosity. The box plots in Figure $3 \mathrm{c}$ show that more than $90 \%$ of the vinegars assigned to the quality category $\mathrm{F}$ were characterized by lowest levels of yield stress and more homogeneous flow, i.e. $\mathrm{R}(1,2)$ was on average very close to 1 , so that it must be concluded that colloidal jamming negatively affected the decision of the judges prevailing on the overall olfactive and gustative properties. Judges assigned the best scores to the vinegars with higher shear-thinning traits at the sub-colloidal scale rather than shearthickening ones (Figure 3d).

\section{Supervised Analysis of Data}

A descriptive analysis of the results from perceptual assessment of the sensory properties was investigated accounting for both the composition and rheological properties following a supervised approach, i.e. assuming the prior knowledge about which vinegar samples belonged to one of the two quality categories, F and R. Since standard descriptive statistics from Shapiro-Wilks' $\mathrm{W}$ and Lilliefors tests highlighted asymmetric distribution for the most of the vinegar properties (data not shown), the Kolmogorov-Smirnov two-sample test, which is based on cumulative distribution, was preferred to summarize differences among the vinegars. The most significant differences between $\mathrm{F}$ and $\mathrm{R}$ quality categories were calculated at $\mathrm{p}<0.05$ for the dynamic viscosities measured at $10 \mathrm{~s}^{-1}, 60 \mathrm{~s}^{-1}, 600 \mathrm{~s}^{-1}$, V600/Density as well as for Brix degree, titratable acidity, and volatile acidity (Table 2). Vinegar samples belonging to the quality category $\mathrm{F}$ were characterized on average by viscosity levels more than double those of vinegars assigned to the category R. No significant differences were observed for sugars, density and fixed acidity. Composition and rheological properties were also used as independent variables, while quality category ( $\mathrm{F}$ and $\mathrm{F}$ ) as grouping variable for the principal component analysis (PCA). Results from PCA are summarized using the plot of scores and variables (Figure 4). A scree plot was used to graphically determine the optimal number of factors to retain. The original variables clustered in two new meaningful, independent variables that could be described respectively as "Homogeneous vs Heterogeneous Flow" according to the factor loading on Factor 1 and as "Overall solute concentration to volatile compounds Balances" according to the factor loading on Factor 2 . Factor 1 accounted for the $61.73 \%$ of the experimental variance, with the dynamic viscosities and kinematic viscosity oppositely related to colloidal jamming property. Factor 2 accounted for about $23.05 \%$ of the experimental variance, with the ratio between Brix degree and titratable acidity oppositely related to the ratio between the volatile acidity and titratable acidity. 
104 | Falcone et al.

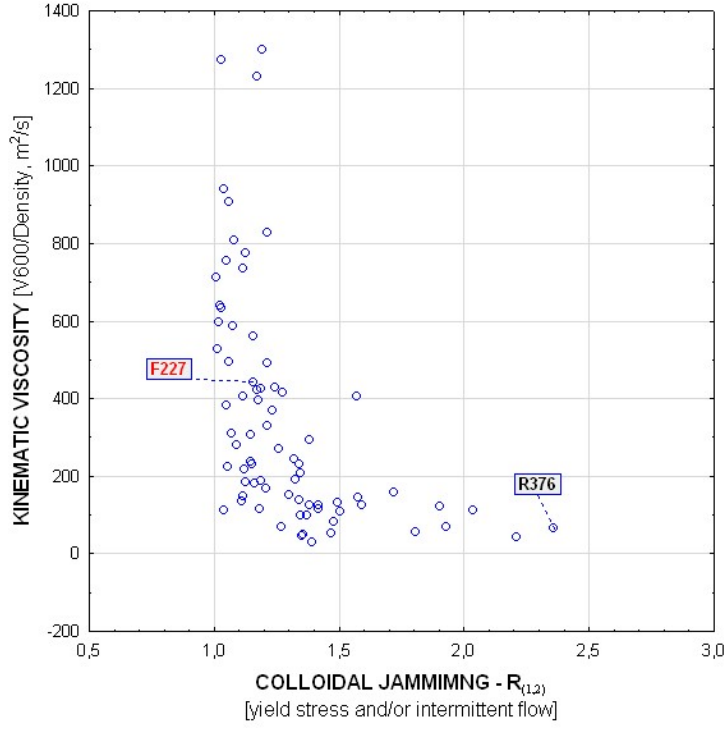

(A)

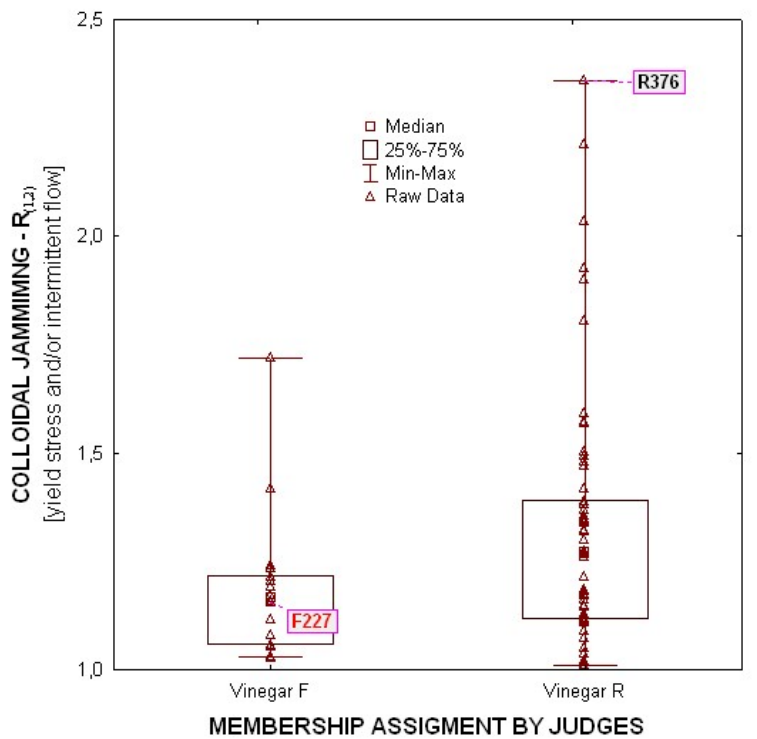

(C)

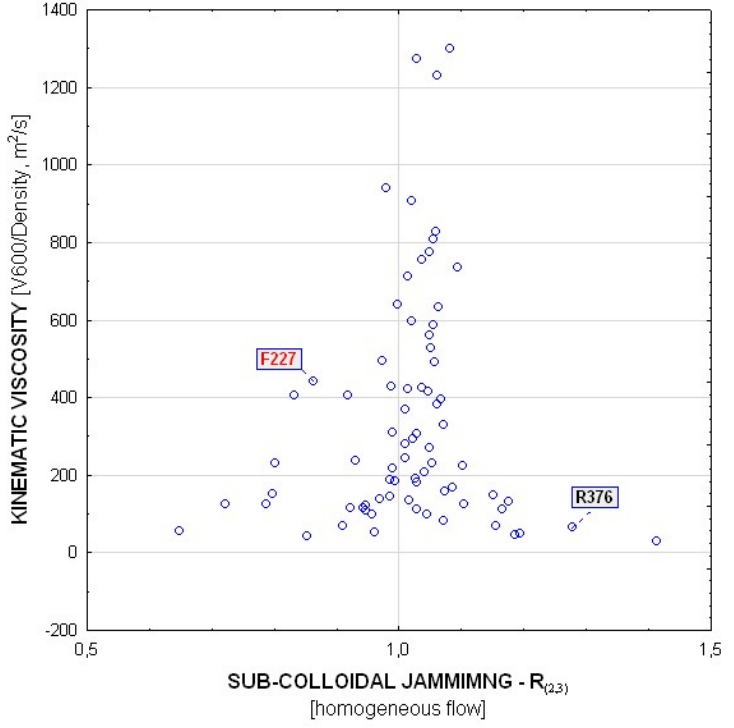

(B)

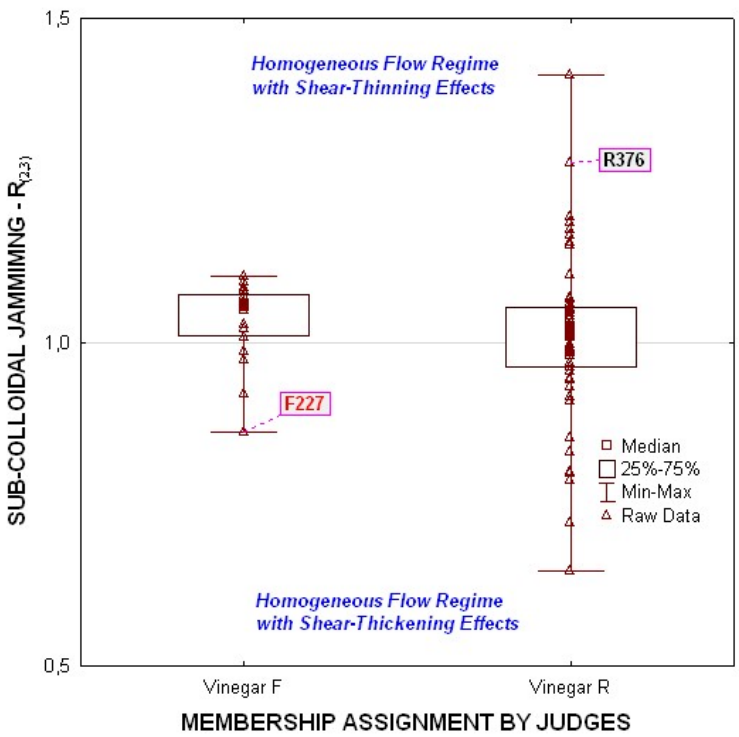

(D)

Figure 3: Distribution of TBVRE samples as function of kinematic viscosity measured at $600 \mathrm{~s}^{-1}$ against the extent of colloidal (a) and sub-colloidal jamming (b). Membership of the vinegars to the two quality categories F, i.e. "aged vinegars of very-high sensory quality" and R, i.e. "aged vinegars of acceptable sensory quality" was affected by the colloidal (c) and sub-colloidal jamming (d). The rheological marker $\mathrm{R}_{(1,2)}$ was associated with the mechanical stimuli of the in-mouth perception of colloidal jamming, while $\mathrm{R}_{(2,3)}$ to the mechanical stimuli of the in-mouth perception of sub-colloidal jamming 
New Quality Markers for Traditional Balsamic Vinegar | 105

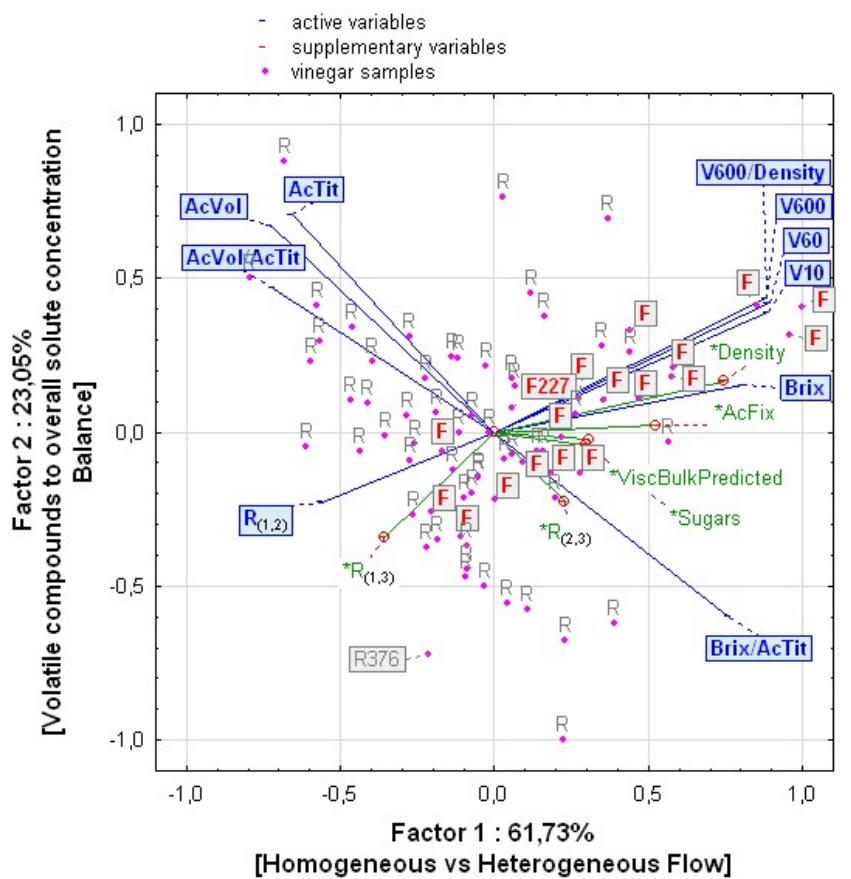

(A)
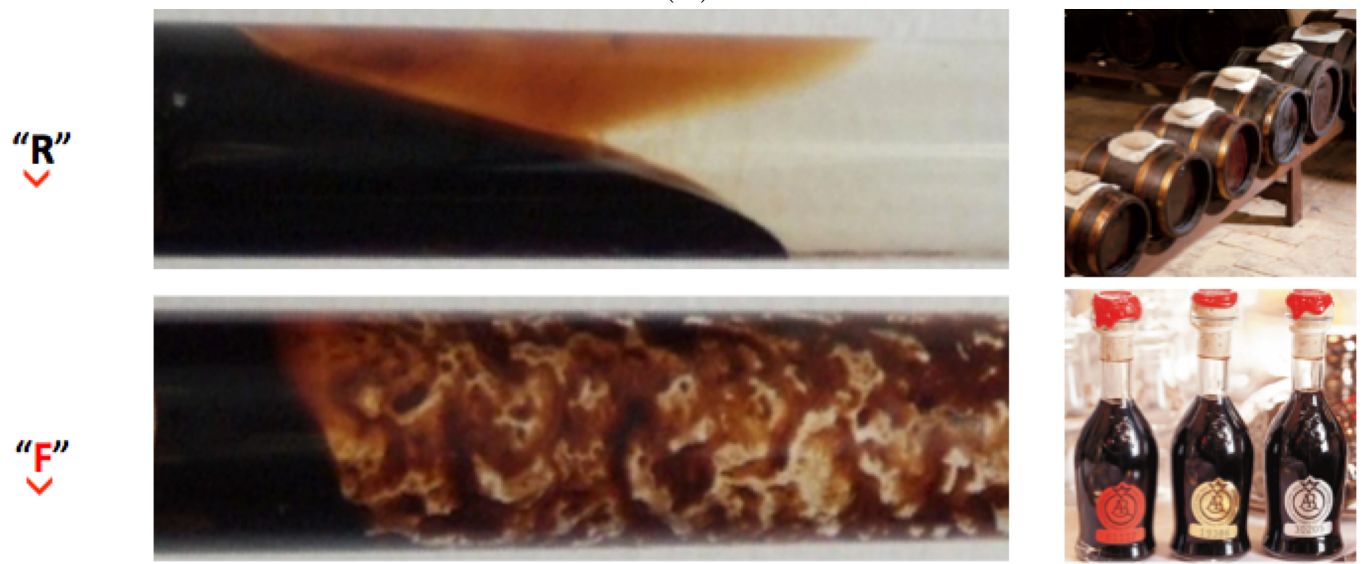

$\begin{array}{ll}F & \text { Aged vinegars of very-high sensory quality as perceived according to the official method } \\ R & \text { Aged vinegars of typical sensory quality as perceived according to the official method } \\ R_{1,2} & \text { Ratio between the apparent viscosity measured at about } 10 \mathrm{~s}^{-1} \text { and that measured at } 60 \mathrm{~s}^{-1} \\ \text { V10, V60, V600 } & \text { Apparent viscosity measured at the targeted shear rates around } 10 \mathrm{~s}^{-1}, 60 \mathrm{~s}^{-1}, 600 \mathrm{~s}^{-1} \\ \text { V600/Density } & \text { kinematic viscosity } \\ \text { AcVol } & \text { Volatile acidity } \\ \text { AcTit } & \text { Titratable acidity } \\ \text { Brix } & \text { Brix degree }\end{array}$

Figure 4: Results from supervised Principal Component Analysis showing distribution of the vinegars with respect to their rheological and composition properties (a). (b) the visual perception of the flow regime under gravity as perceived according to the official sensory method for vinegars belonging to the quality category "R" (intermittent flow) or to the quality category "F" (homogeneous flow) 
Table 2: Results from Kolmogorov-Smirnov two-sample test used to assess the differences between the two quality categories of vinegars R, i.e. the "aged vinegars of acceptable sensory quality" and F i.e. the "aged vinegars of very-high sensory quality" in terms of composition and rheological properties. Marked tests are significant at $\mathrm{p}<0.005$

\begin{tabular}{llllllll}
\hline VAR & $\begin{array}{l}\text { Max Negative } \\
\text { Difference }\end{array}$ & $\begin{array}{l}\text { Max Positive } \\
\text { Difference }\end{array}$ & $\mathrm{p}$-value & $\begin{array}{l}\text { Mean } \\
\mathrm{F}\end{array}$ & $\begin{array}{l}\text { Mean } \\
\mathrm{R}\end{array}$ & $\begin{array}{l}\text { Std. Dev. } \\
\mathrm{F}\end{array}$ & $\begin{array}{l}\text { Std. Dev. } \\
\mathrm{R}\end{array}$ \\
\hline Sugars & -0.105 & 0.268 & $\mathrm{P}>0.100$ & 43.302 & 41.961 & 2.037 & 3.351 \\
Brix & 0.000 & 0.391 & $\mathrm{P}<0.050$ & 71.817 & 67.945 & 3.076 & 4.961 \\
Density & 0.000 & 0.341 & $\mathrm{P}<0.100$ & 1.359 & 1.339 & 0.021 & 0.030 \\
AcTit & -0.471 & 0.155 & $\mathrm{P}<0.005$ & 5.027 & 6.116 & 0.473 & 1.772 \\
AcFix & 0.000 & 0.335 & $\mathrm{P}<0.100$ & 1.505 & 1.280 & 0.295 & 0.323 \\
AcVol & -0.506 & 0.052 & $\mathrm{P}<0.005$ & 3.522 & 4.836 & 0.612 & 1.822 \\
V10 & 0.000 & 0.536 & $\mathrm{P}<0,001$ & 1010.000 & 426.579 & 623.411 & 303.806 \\
V60 & 0.000 & 0.536 & $\mathrm{P}<0.001$ & 883.648 & 356.979 & 559.863 & 296.951 \\
V600 & 0.000 & 0.584 & $\mathrm{P}<0.001$ & 848.493 & 353.573 & 522.643 & 290.431 \\
$\mathrm{R}_{(1,2)}$ & -0.423 & 0.068 & $\mathrm{P}<0.025$ & 1.187 & 1.327 & 0.165 & 0.296 \\
$\mathrm{R}_{(2,3)}$ & -0.155 & 0.331 & $\mathrm{P}<0.100$ & 1.032 & 1.011 & 0.063 & 0.125 \\
$\mathrm{R}_{(1,3)}$ & -0.341 & 0.094 & $\mathrm{P}<0.100$ & 1.225 & 1.341 & 0.184 & 0.359 \\
ViscBlukPredicted & -0.105 & 0.268 & $\mathrm{P}<0.100$ & 0.002 & 0.002 & 0.000 & 0.000 \\
Brix/AcTit & -0.068 & 0.506 & $\mathrm{P}<0.005$ & 14.430 & 12.034 & 1.731 & 3.533 \\
V600/Density & 0.000 & 0.584 & $\mathrm{P}<0.001$ & 620.888 & 260.888 & 378.291 & 210.395 \\
\hline
\end{tabular}

As can be inferred from PCA results, flow properties dominated over the composition ones underpinning the perceptual classification of sensory properties by judges towards vinegars showing lower but more homogeneous flowiness with a very narrow distribution centered on the mean level for the compositional balance (where the Factor 2 is equal to 0 ), i.e. with respect for both the overall solute concentration (including sugars and titratable acidity) and volatile acidity (including acetic acid). Such a compositional balance was thought as an optimum for such aged vinegars. It is worth noting that the official tasting procedure for TBVRE imposes different coefficients for weight contribution from visual, olfactive, and gustative perceptions, reserving the lower one (i.e. 15\%) for the visual perception of the vinegar flowiness and light transparency. As a consequence, it was concluded that the extent of jamming at the colloidal scale plays a strong masking effect on the perceptual intensity of both olfactory and gustatory sensing.

\section{Unsupervised Analysis of Data}

An important key point is the fact that the sensory score assigned by the panellists were the result of a top-down decision process consisting of a series of judgments related to their perception of more than one sensory attribute at a time. The official tasting procedure, in fact, imposes a series of ordered, stepwise evaluation tests for coded visual, olfactory and taste attributes. Without any a priori hypotheses about how many independent quality categories as well as about which vinegar sample may belong to each of them, tree classification analysis (TCA) was carried out based on classification, splitting and validation rules examining the effects of the predictor variables one at a time, rather than just all at once. The top-down induction process started by dividing the initial set of vinegars into subsets in a hierarchical manner, using a splitting value test for one composition or one rheological property at time as target variable. Such recursive partitioning was completed when splitting no longer added value to the predictions, i.e. when no further vinegar was assigned to the same cluster of the tree. The classification rule 
New Quality Markers for Traditional Balsamic Vinegar | 107

Table 3: Tree structure from unsupervised classification tree analysis for composition and rheological related properties as predictor variables one at once, encoding for the top-down decision process followed for vinegar classification according to the official sensory procedure

\begin{tabular}{ccccccccc}
\hline NODE & $\begin{array}{c}\text { Left } \\
\text { Branch }\end{array}$ & $\begin{array}{c}\text { Right } \\
\text { Branch }\end{array}$ & $\begin{array}{c}\text { Size of } \\
\text { Node }\end{array}$ & $\begin{array}{c}\mathrm{N} \text { in } \\
\text { class F }\end{array}$ & $\begin{array}{c}\text { N in } \\
\text { class R }\end{array}$ & $\begin{array}{c}\text { Selected } \\
\text { Category }\end{array}$ & $\begin{array}{c}\text { Split } \\
\text { Variable }\end{array}$ & $\begin{array}{c}\text { Split } \\
\text { Constant }\end{array}$ \\
\hline 1 & 2 & 3 & 75 & 18 & 57 & $\mathrm{R}$ & V600/Density & 426.5 \\
2 & 4 & 5 & 54 & 5 & 49 & $\mathrm{R}$ & $\mathrm{R}_{(1,2)}$ & 1,1 \\
4 & 6 & 7 & 42 & 2 & 40 & $\mathrm{R}$ & Brix/AcTit & 12.5 \\
6 & & & 24 & 0 & 24 & $\mathrm{R}$ & & \\
7 & 8 & 9 & 18 & 2 & 16 & $\mathrm{R}$ & AcVol/AcTit & 0.8 \\
8 & 10 & 11 & 17 & 1 & 16 & $\mathrm{R}$ & V600/Density & 349.6 \\
10 & & & 13 & 0 & 13 & $\mathrm{R}$ & & \\
11 & 12 & 13 & 4 & 1 & 3 & $\mathrm{R}$ & V600/Density & 376.2 \\
12 & & & 1 & 1 & 0 & $\mathrm{~F}$ & & \\
13 & & & 3 & 0 & 3 & $\mathrm{R}$ & & \\
9 & & & 1 & 1 & 0 & $\mathrm{~F}$ & & \\
5 & 14 & 15 & 12 & 3 & 9 & $\mathrm{R}$ & V600/Density & 152.7 \\
14 & & & 9 & 0 & 9 & $\mathrm{~F}$ & & \\
15 & & & 3 & 3 & 0 & $\mathrm{~F}$ & & \\
3 & 16 & 17 & 21 & 13 & 8 & $\mathrm{~F}$ & AcVol/AcTit & 0.7 \\
16 & 18 & 19 & 12 & 11 & 1 & $\mathrm{~F}$ & Brix/AcTit & 18.1 \\
18 & & & 11 & 11 & 0 & $\mathrm{~F}$ & & \\
19 & & & 1 & 0 & 1 & $\mathrm{R}$ & & \\
17 & 20 & 23 & 3 & 2 & 1 & $\mathrm{~F}$ & R & \\
20 & & & 6 & 0 & 6 & $\mathrm{R}$ & & 1.1 \\
21 & 22 & 23 & 3 & 2 & 1 & $\mathrm{~F}$ & Brix/AcTit & 14.3 \\
22 & & & 2 & 2 & 0 & $\mathrm{~F}$ & & \\
23 & & & 1 & 0 & 1 & $\mathrm{R}$ & & \\
\hline
\end{tabular}

Table 4: Misclassification matrix from unsupervised classification tree analysis accounting for composition and composition related properties as predictor variables one at once, encoding for the top-down decision process followed for vinegar classification according to the official sensory procedure

\begin{tabular}{lllll}
\hline & OBSERVED & PREDICTED F & PREDICTED R & Row Total \\
\hline Number & $\mathrm{F}$ & 18 & & 18 \\
Column Percentage & & $100.00 \%$ & $0.00 \%$ & \\
Row Percentage & & $100.00 \%$ & $0.00 \%$ & \\
Total percentage & $\mathrm{R}$ & $23.68 \%$ & $0.00 \%$ & $23.68 \%$ \\
$\begin{array}{l}\text { Number } \\
\text { Column percentage }\end{array}$ & & $0.00 \%$ & 58 & 58 \\
Row Percentage & & $0.00 \%$ & $100.00 \%$ & \\
Total percentage & & $0.00 \%$ & $76.00 \%$ & $76.32 \%$ \\
Count & All Group & 18 & $58 \%$ & 76 \\
TOTAL PERCENT & & & 2 & 2 \\
\hline
\end{tabular}




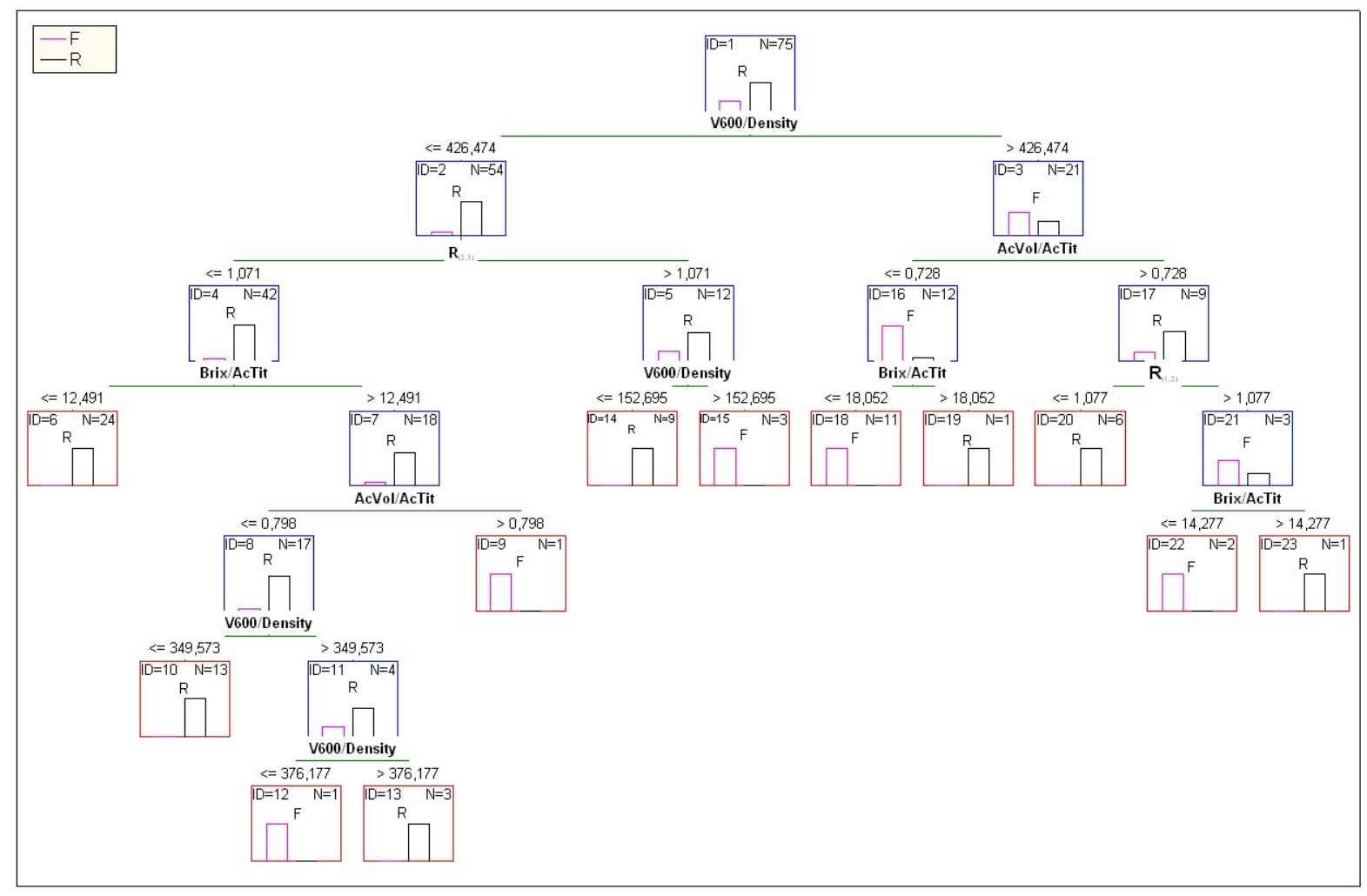

Figure 5: Tree graph showing results from unsupervised classification tree analysis accounting for rheological and composition properties as predictor variables one at a time, encoding for the top-down decision process followed for vinegar classification according to the official sensory procedure. The number of terminal nodes was 12

was to assign membership of vinegars to the $\mathrm{F}$ or $\mathrm{R}$ category with the same accuracy, regardless of the frequency really assigned by the judges for the same categories. Corroborating results from PCA, results from TCA suggest a pattern of recognition for vinegar quality relying mostly on V600/Density and $\mathrm{R}(1,2)$ but with a minor contribution, it relies on the balance between the overall solute concentration and volatile compounds. Figure 5 shows the tree graph from unsupervised tree analysis accounting for structure and composition properties as predictor variables one at a time, obtained without misclassification costs. Tree structure data are shown in Table 3 , while misclassification costs are given in Table 4. Data reported in Figure 6 together with those from the misclassification matrix (Table 4) are evidence of the goodness-of-fit of the classification tree. As can be inferred from Table 3, V600/Density was the best parameter to split locally the starting set of vinegar samples (426.5 Pa.s was the split value test). The first major sub-group consisted of 54 vinegars, the $90 \%$ of which were vinegars belonging to the R category, and could be divided into two subsets, where the $\mathrm{R}(2,3)$ was the locally optimal decision predictor and 1.10 the split value test. The second major sub-group, consisting of 21 vinegars, $60 \%$ of which vinegars belonging to the F category, could be divided in two subsets, based on the ratio between volatile acidity and titratable acidity (0.73 split value test). This could be continued throughout the entire tree decision process until the terminal nodes were reached where one could 


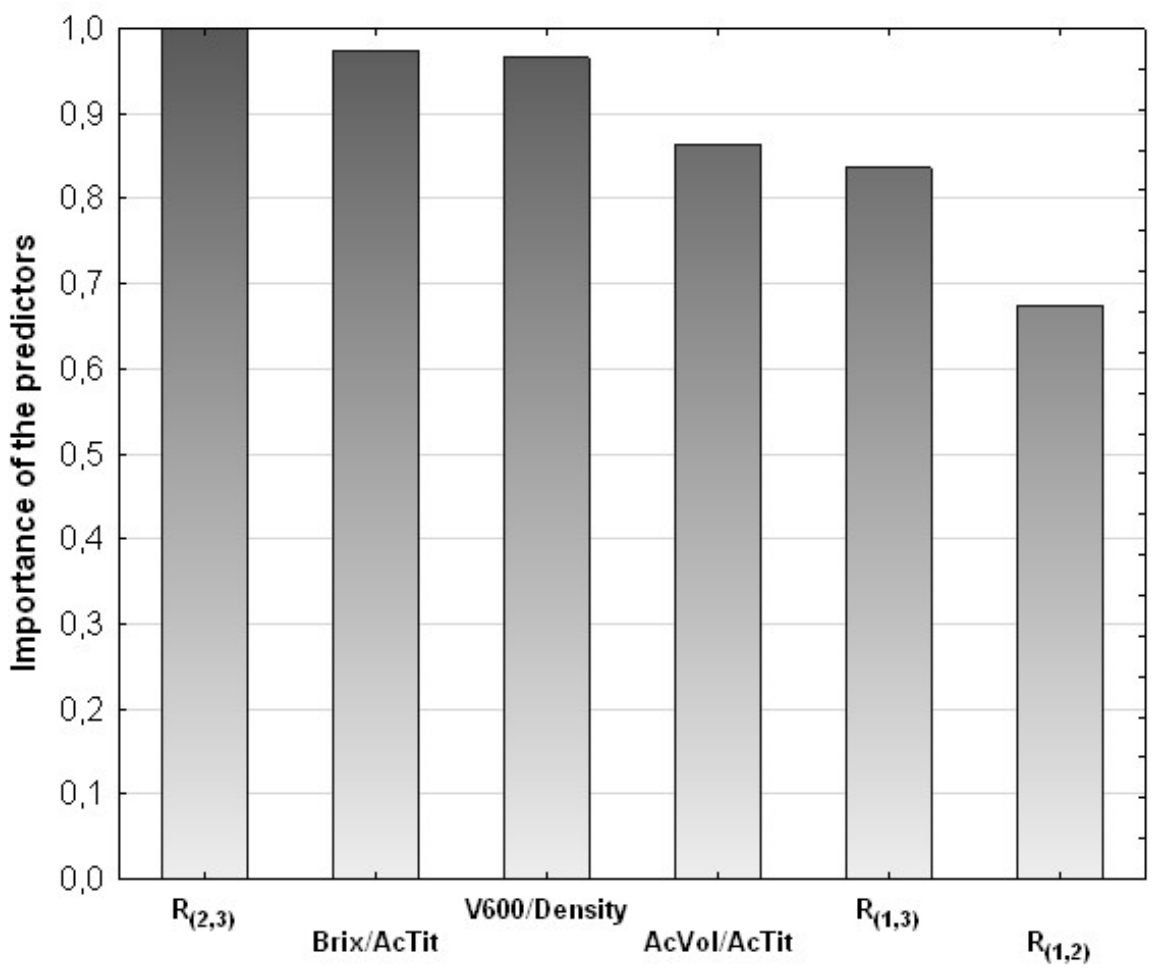

Figure 6: Importance plot from unsupervised classification tree analysis accounting for structure- and composition properties as predictor variables one at a time, encoding for the top-down decision process followed for vinegar classification according to the official sensory procedure

observe the final membership predictions of the vinegars within one of the two quality categories. As can be inferred from data, the relative importance of prediction variables that could be associated to the judges assignation followed a decreasing order for the descriptor of sub-colloidal jamming, i.e. $\mathrm{R}(2,3)$, the ratio between Brix degree and total acidity, and the kinematic viscosity. Such results suggested that the final score assigned by the panellists may have been the result of "reverse type" top-down decision process, where they provided a series of judgments aiming unconsciously to classify vinegars with respect to their perception of jamming properties and then with respect for their composition-related perception.

\section{Conclusions}

It is our strong belief that before the assignment of the Traditional Balsamic Vinegar designation, the official regulations must require compliance with the dynamic viscosity as a new characteristic marker of the quality of the vinegar together with the corresponding instrumental method to be followed for its measurement under controlled shearing conditions.

In this work, two different flow regimes were clearly detected among the vinegars: (i) an intermittent or heterogeneous flow at the lowest shear rates; (ii) a homogeneous and continuous flow regime at medium-high shear rates showing some shear thickening and/or thinning traits. Physical and structural insights of colloidal jamming on flow behaviour were gained, while the physical origin of the flow intermittency was attributed to a change in nature of the interactions between 
vinegar melanoidins at a colloidal scale due to the mechanical shear. Over a critical shear rate, the apparent shear stress induced phase transition from the intermittent to homogeneous flow regime. Our study provided evidence that the extent of jamming at sub-colloidal scale plays a strong masking effect on the overall olfactory and gustatory perceptions. Jamming properties at sub-colloidal scale affected the perceptual classification of sensory properties towards vinegars showing lower but more homogeneous flow, with a very narrow distribution against the optimum of balance between the total solute concentration (including sugars and acidity) and volatile compounds. A classification tree model able to describe the top-down decision process for membership assignment according to the official tasting procedure was developed and validated accounting for composition and rheological properties separately, rather than just all at once. Results suggested that the perceptual assessment of the sensorial properties of the vinegar, as performed by the trained judges according to the official sensory procedure, relies mostly on colloidal jamming and kinematic viscosity measured at $600 \mathrm{~s}^{-1}$ and less so on the balance between total solids and volatile acidity as well as on the balance between volatile acidity and total acidity.

\section{References}

Akhtar, M., Stenzel, J., Murray, B. S., \& Dickinson, E. (2005). Factors affecting the perception of creaminess of oil-in-water emulsions. Food Hydrocolloids, 19(3), 521-526. Conference on Food Colloids, Harrogate, ENGLAND, APR 18-21, 2004. doi:10.1016/j . foodhyd.2004.10.017

Boquet, R., Chirife, J., \& Iglesias, H. A. (1978). Equations for fitting water sorption isotherms of foods .2. evaluation of various 2-parameter models. Journal of Food Technology, 13(4), 319-327.

Camacho, S., Dop, M., de Graaf, C., \& Stieger, M. (2015). Just noticeable differences and weber fraction of oral thickness perception of model beverages. Journal of Food Sci- ence, 80(7), S1583-S1588. doi:10.1111/ 1750-3841.12922

Cates, M. E., Wittmer, J. P., Bouchaud, J. P., \& Claudin, P. (1998). Jamming, force chains, and fragile matter. Physical Review Letters, 81(9), 1841-1844. doi:10.1103/ PhysRevLett.81.1841

Chen, J. \& Engelen, L. (2012). Food oral processing: fundamentals of eating and sensory perception. John Wiley \& Sons.

Cocchi, M., Durante, C., Grandi, M., Lambertini, P., Manzini, D., \& Marchetti, A. (2006). Simultaneous determination of sugars and organic acids in aged vinegars and chemometric data analysis. Talanta, 69(5), 1166-1175. doi:10.1016/j.talanta.2005.12. 032

Cook, D. J., Hollowood, T. A. ., Linforth, R. S. T., \& Taylor, A. J. (2003). Oral shear stress predicts flavour perception in viscous solutions. Chemical Senses, 28(1), 11-23. doi:10.1093/chemse/28.1.11

Coussot, P. \& Ovarlez, G. (2010). Physical origin of shear-banding in jammed systems. European Physical Journal E, 33(3), 183188. doi:10.1140/epje/i2010-10660-9

Duran Guerrero, E., Chinnici, F., Natali, N., Natera Marin, R., \& Riponi, C. (2008). Solid-phase extraction method for determination of volatile compounds in traditional balsamic vinegar. Journal of Separation Science, 31(16-17), 3030-3036. doi:10. $1002 /$ jssc. 200800307

Falcone, P. M. (2010). Crystallization and jamming in the traditional balsamic vinegar. Food Research International, 43(8), 22172220. doi:10.1016/j.foodres.2010.07.013

Falcone, P. M., Boselli, E., \& Frega, N. G. (2011). Structure-composition relationships of the traditional balsamic vinegar close to jamming transition. Food Research International, 44(6), 1613-1619. doi:10.1016/j . foodres.2011.04.028

Falcone, P. M., Chillo, S., Giudici, P., \& Del Nobile, M. A. (2007). Measuring rheological properties for applications in quality assessment of traditional balsamic vinegar: description and preliminary evaluation of a model. Journal of Food Engineering, 80(1), 
234-240. doi:10.1016/j.jfoodeng.2006.05. 023

Falcone, P. M. \& Giudici, P. (2008). Molecular size and molecular size distribution affecting traditional balsamic vinegar aging. Journal of Agricultural and Food Chemistry, 56(16), 7057-7066. doi:10 . 1021/ jf800706g

Falcone, P. M., Mozzon, M., \& Frega, N. G. (2012). Structure-composition relationships of the traditional balsamic vinegar of modena close to jamming transition (part ii): threshold control parameters. Food Research International, 45(1), 75-84. doi:10.1016/j.foodres.2011.09.003

Falcone, P. M., Verzelloni, E., Tagliazucchi, D., \& Giudici, P. (2008). A rheological approach to the quantitative assessment of traditional balsamic vinegar quality. Journal of Food Engineering, 86(3), 433-443. doi:10.1016/j.jfoodeng.2007.10.017

Gebhard, S. (2000). A practical approach to rheology and rheometry. Gebrueder HAAKE GmbH, D-76227 Karlsruhe, Dieselstrasse, 4.

Giudici, P., Falcone, P. M., Scacco, A., \& Lanza, C. M. (2009). Sensory analysis of the traditional balsamic vinegar. Industrie delle Bevande, 38(224), 27-49.

Hollowood, T. A., Linforth, R. S. T., \& Taylor, A. J. (2002). The effect of viscosity on the perception of flavour. Chemical Senses, 27(7), 583-591. doi:10.1093/chemse/27.7. 583

Holmes, W. M., Callaghan, P. T., Vlassopoulos, D., \& Roovers, J. (2004). Shear banding phenomena in ultrasoft colloidal glasses. Journal of Rheology, 48(5), 1085-1102. doi:10.1122/1.1773829

Krieger, I. M. \& Dougherty, T. J. (1959). A mechanism for non-newtonian flow in suspensions of rigid spheres. Transactions of the Society of Rheology, 3(1), 137-152.

Lemmetti, F., Falcone, P. M., \& Giudici, P. (2013). Traditional balsamic vinegar: the role of the viscosity on the objective and perceived quality. Industr Bevande, 244, 921.

Lemmetti, F., Solieri, L., Bonciani, T., Zanichelli, G., \& Giudici, P. (2014). Sensory analy- sis of traditional balsamic vinegars: current state and future perspectives. Acetic Acid Bacteria, 3(1).

Liu, A. J. \& Nagel, S. R. (1998). Nonlinear dynamics: jamming is not just cool any more. Nature, 396(6706), 21-22. doi:10 . 1038/ 23819

Liu, A. J. \& Nagel, S. R. (2001). Jamming and rheology: constrained dynamics on microscopic and macroscopic scales. CRC Press.

Mezzenga, R., Schurtenberger, P., Burbidge, A., \& Michel, M. (2005). Understanding foods as soft materials. Nature Materials, 4(10), 729-740. doi:10.1038/nmat1496

Plessi, M., Monzani, A., \& Coppini, D. (1988). Determination of the monosaccharide and alcohol content of balsamic and other vinegars by enzymatic methods. Agricultural and Biological Chemistry, 52(1), 25-30.

Princen, H. M. \& Kiss, A. D. (1989). Rheology of foams and highly concentrated emulsions .4. an experimental-study of the shear viscosity and yield stress of concentrated emulsions. Journal of Colloid and Interface Science, 128(1), 176-187. doi:10.1016/ 0021-9797(89)90396-2

Schall, P. \& van Hecke, M. (2010). Shear bands in matter with granularity. Annual Review of Fluid Mechanics. Annual Review of Fluid Mechanics, 42, 67-88. doi:10.1146/ annurev-fluid-121108-145544

Shama, F., Parkinson, C., \& Sherman, P. (1973). Identification of stimuli controlling the sensory evaluation of viscosity i. non-oral methods. Journal of Texture Studies, 4(1), 102-110. doi:10.1111/j. 1745-4603. 1973. tb00656.x

Tagliazucchi, D., Verzelloni, E., \& Conte, A. (2010). Contribution of melanoidins to the antioxidant activity of traditional balsamic vinegar during aging. Journal of Food Biochemistry, 34(5), 1061-1078. doi:10.1111/ j.1745-4514.2010.00349.x

Trappe, V., Prasad, V., Cipelletti, L., Segre, P. N., \& Weitz, D. A. (2001). Jamming phase diagram for attractive particles. $\mathrm{Na}$ ture, 411(6839), 772-775. doi:10.1038/ 35081021

Trulsson, M. \& Essick, G. K. (1997). Lowthreshold mechanoreceptive afferents in the 
human lingual nerve. Journal of Neurophysiology, $77(2), 737-748$.

Ubbink, J., Burbidge, A., \& Mezzenga, R. (2008). Food structure and functionality: a soft matter perspective. Soft Matter, 4(8), 1569-1581. doi:10.1039/b802183j

Verzelloni, E., Tagliazucchi, D., \& Conte, A. (2007). Relationship between the antioxidant properties and the phenolic and flavonoid content in traditional balsamic vinegar. Food Chemistry, 105(2), 564-571. doi:10.1016/j.foodchem.2007.04.014

Vingerhoeds, M. H., de Wijk, R. A., Zoet, F. D., Nixdorf, R. R., \& van Aken, G. A. (2008). How emulsion composition and structure affect sensory perception of lowviscosity model emulsions. Food Hydrocolloids, 22(4), 631-646. doi:10 . 1016 / j . foodhyd.2007.02.011

Wood, F. W. (1968). Psychophysical studies on the consistency of liquid foods. Rheology and Texture of Food Stuffs, SCI Monograph, (27), 40-49.

Zahn, S., Hoppert, K., Ullrich, F., \& Rohm, H. (2013). Dairy-based emulsions: viscosity affects fat difference thresholds and sweetness perception. Foods, 2(4), 521-533. doi:10.3390/foods2040521 